\title{
ZENK induction in the zebra finch brain by song: Relationship to hemisphere, rhythm, oestradiol and sex
}

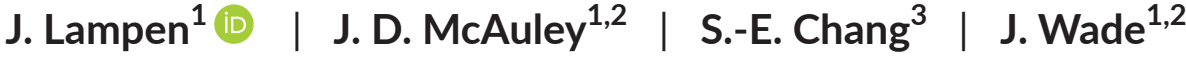

${ }^{1}$ Neuroscience Program, Michigan State University, East Lansing, MI, USA

${ }^{2}$ Department of Psychology, Michigan State University, East Lansing, MI, USA

${ }^{3}$ Department of Psychiatry, University of Michigan, Ann Arbor, MI, USA

\section{Correspondence}

Jennifer Lampen, Neuroscience Program, Michigan State University, East Lansing, MI, USA.

Email: lampenje@msu.edu

\section{Funding information}

National Institutes of Health, Grant/Award Number: R01-MH096705; Michigan State University's program for Research in Autism, Intellectual and Neurodevelopmental

Disabilities (RAIND)
Oestradiol is abundant in the zebra finch auditory forebrain and has the capacity to modulate neural responses to auditory stimuli with specificity as a result of both hemisphere and sex. Arrhythmic song induces greater ZENK expression than rhythmic song in the caudomedial nidopallium (NCM), caudomedial mesopallium (CMM) and nucleus taeniae ( $\mathrm{Tn}$ ) of adult zebra finches. The increases in the auditory regions (i.e. NCM and CMM) may result from detection of errors in the arrhythmic song relative to the learned template. In the present study, zebra finches were treated with oestradiol, the aromatase inhibitor fadrozole or a control and then exposed to rhythmic or arrhythmic song to assess the effect of oestradiol availability on neural responses to auditory rhythms. ZENK mRNA was significantly greater in the left hemisphere within the NCM, CMM and Tn. Main effects of sex were detected in both auditory regions, with increased ZENK in males in the NCM and in females in the CMM. In the CMM, an effect of hormone treatment also existed. Although no pairwise comparison was statistically significant, the pattern suggested greater ZENK expression in control compared to both fadrozole- and oestradiol-treated birds. In the NCM, an interaction between sex and hormone treatment suggested that the sex effect was restricted to control animals. An additional interaction in the NCM among sex, stimulus rhythmicity and hemisphere indicated that the strongest effect of laterality was present in males exposed to arrhythmic song. The hormone effects suggest that an optimal level of oestradiol may exist for processing rhythmicity of auditory stimuli. The overall pattern for left lateralisation parallels the left lateralisation of language processing in humans and may suggest that this hemisphere is specialised for processing conspecific vocalisations. The reversed sex differences in the NCM and CMM suggest that males and females differentially rely on components of the auditory forebrain for processing conspecific song.

\section{KEYWORDS}

caudomedial mesopallium, caudomedial nidopallium, Egr-1, lateralisation, nucleus taeniae, songbird

\section{1 | INTRODUCTION}

Oestradiol $\left(E_{2}\right)$ influences perceptual systems in a large range of animals. The hormone may act at both peripheral and central levels. For example, oestrogen receptor $\alpha$ is present in the retina, ${ }^{1-3}$ and oestrogen receptors $\alpha$ and $\beta$ are present in many sensory organs, including the olfactory epithelium, ${ }^{4}$ dorsal root ganglion and cochlea ${ }^{5-7}$ in diverse species. Women with high levels of $E_{2}$ show attenuation of auditory event-related potentials in the cortex, ${ }^{8}$ and auditory evoked responses have reduced latencies in the left hemisphere during high $E_{2}$ 
phases of the menstrual cycle. ${ }^{9}$ Auditory brainstem responses are also influenced by the $E_{2}$ levels in rats, ${ }^{10}$ rhesus monkeys ${ }^{11}$ and humans. ${ }^{12}$

In zebra finches, the caudomedial nidopallium (NCM), a secondary auditory cortical region, and the nucleus taeniae $(\mathrm{Tn})$, a region of the avian brain that appears analogous to the mammalian medial amygdala, ${ }^{13}$ are sites of substantial $E_{2}$ synthesis and activity. ${ }^{14,15}$ Both areas have abundant expression of aromatase, ${ }^{16-18}$ the enzyme responsible for the metabolism of $E_{2}$ from testosterone. The NCM and Tn and, to a lesser degree, the caudomedial mesopallium (CMM), another secondary auditory region, express both oestrogen receptor $\alpha^{19,20}$ and the membrane bound receptor G-protein coupled receptor 30 (GPR30). ${ }^{21}$ Microdialysis in the NCM of awake behaving zebra finches reveals a significant increase in $\mathrm{E}_{2}$ concentration during exposure to conspecific song in both males ${ }^{22}$ and females. ${ }^{23}$

The effects of $E_{2}$ on auditory discrimination in songbirds have been assessed through multiple methodologies. Electrophysiological recordings within the zebra finch NCM with simultaneous retrodialysis of $E_{2}$ showed consistently increased neural responses to conspecific songs. ${ }^{23-25}$ The pattern is complicated, however, by increased responses to white noise detected in some studies but not others, ${ }^{23-25}$ as well as retrodialysis of fadrozole (a potent aromatase inhibitor) reducing overall responses to sound in one study but not another. ${ }^{23,24,26}$ Thus, although $E_{2}$ within the NCM appears to increase responses to conspecific song, its exact influence on auditory selectivity in this region is unclear. In seasonally breeding white-throated sparrows, no differences exist in expression of the immediate early gene ZENK in the NCM or CMM of nonbreeding individuals between exposure to conspecific song or tones. However, with systemic $E_{2}$ replacement, higher ZENK is seen in birds exposed to song. ${ }^{27}$ These effects are not the result of an increase in ZENK in response to conspecific song but, instead, to a decrease in response to tones. ${ }^{27}$ Similar patterns of ZENK expression are seen in brain regions within the social behaviour network ${ }^{28}$ including the Tn, where birds in the breeding season have greater ZENK expression in response to song than to tones or silence, and exogenous $E_{2}$ induces this difference in nonbreeding birds. ${ }^{28}$ Taken together, the results of these studies suggest a strong role for $E_{2}$ in auditory processing in songbirds.

Evidence of lateralisation of processing of ecologically relevant auditory stimuli has been seen in both humans and songbirds. Linguistic perception appears consistently left lateralised in humans (29). Although many studies have detected lateral differences in conspecific song processing in zebra finches, the data are less consistent than those seen in humans. Depending on the housing conditions, auditory stimuli employed and technique used to assess neural activity, right or left lateralisation of function have been detected. ${ }^{30-34}$ Thus, although the function of and conditions leading to lateral response specificity in zebra finches remain somewhat unclear, the data do suggest some lateral specialisation of the processing of ecologically relevant auditory stimuli.

A growing body of evidence links rhythmic ability to linguistic skills in humans. In young adults, a positive correlation exists between the ability to process rhythmic sequences and language and literacy skills. ${ }^{35}$ In addition, deficits in rhythm processing have been detected in a range of speech and language disorders, such as dyslexia, ${ }^{36}$ stuttering ${ }^{37}$ and specific language impairment. ${ }^{38,39}$ Clarifying the factors that influence neural processing of rhythm should aid in understanding these and other disorders involving rhythmic processing deficits. As a vocal learning species with a naturally rhythmic song, ${ }^{40}$ the zebra finch represents a strong model for investigating rhythm processing.

Previous research from our group has investigated the effect of the rhythmicity of song on ZENK responses in the NCM, CMM and Tn. ${ }^{41}$ In all three regions, ZENK expression was increased in adult birds exposed to song modified to disrupt the natural temporal structure (arrhythmic) compared to song with the timing unaltered (rhythmic). ${ }^{41}$ Considering data from humans, ${ }^{42-44}$ these results were interpreted in terms of the increased activity in the NCM and CMM potentially indicating errors in arrhythmic song relative to the more natural vocalisations. Increased activity in Tn may reflect a perception of the singer as a poor potential mate. ${ }^{45,46}$

To further understand the mechanisms underlying auditory rhythm discrimination, in the present study, we investigated the potential impacts of $E_{2}$ on neural ZENK responses to rhythmic and arrhythmic song stimuli by both increasing and decreasing $E_{2}$ availability. We tested both male and female zebra finches aiming to determine whether $E_{2}$ influences increased $Z$ ENK responses to rhythmic and arrhythmic songs previously detected in females compared to males in the $\mathrm{CMM} .{ }^{41}$ We also analysed the right and left hemispheres of each bird independently aiming to determine whether any lateralisation of function was present with this auditory perception task. We hypothesised that $E_{2}$ facilitates auditory rhythm discrimination in zebra finches. Based on this hypothesis and our previous results, we predicted that, with no hormone manipulation, greater ZENK expression would be detected in birds exposed to arrhythmic compared to rhythmic song. Furthermore, we expected that this effect would be attenuated by fadrozole treatment and exaggerated by $E_{2}$ treatment.

\section{2 | MATERIALS AND METHODS}

\section{1 | Subjects}

Zebra finches (54 males and 54 females, nine birds in each combination of sex, hormone treatment and song stimulus type; final group sizes presented in Table 1) hatched and were raised in walk-in aviaries $\left(2.5^{\prime} \times 6^{\prime} \times 5^{\prime}\right)$, housing five to seven pairs of adult birds and their offspring. Birds were kept under a 12:12 hours light/dark cycle, and given ad lib. access to water, seed (Kaytee Finch Feed; Chilton, WI, USA), gravel and cuttlebone. The birds received weekly dietary supplementation with spinach, oranges, bread and hard boiled chicken eggs. When birds were a minimum of 90 days of age, they were transferred to walk-in single sex aviaries of the same dimensions housing 30-60 birds and allowed to acclimate to their new housing conditions for at least 10 days. These aviaries maintained auditory and visual contact with birds in the opposite sex aviary, as well as mixed sex aviaries. Birds remained in the single sex aviaries until the start of 
TAB LE 1 ZENK+ cells $\mathrm{mm}^{-2}$ mean \pm SE for the caudomedial nidopallium (NCM), caudomedial mesopallium (CMM) and nucleus taeniae (Tn)

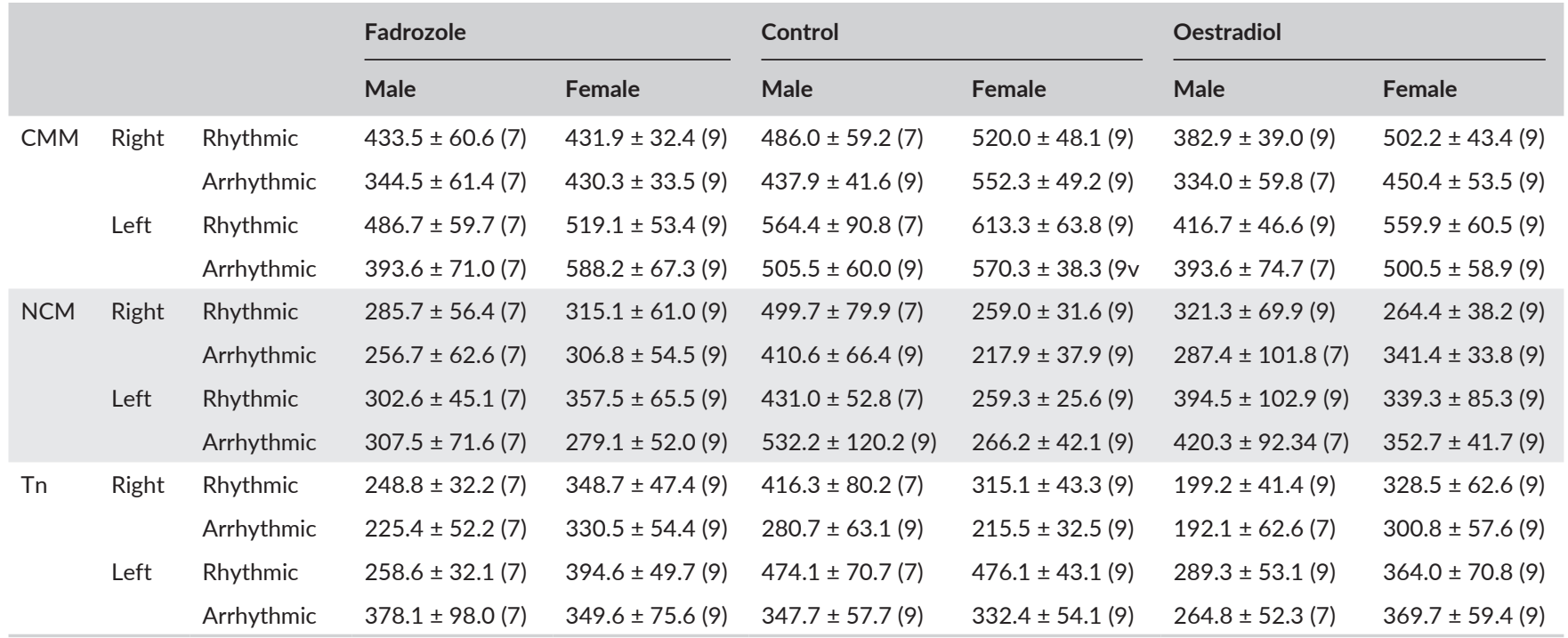

The sample size is indicated in parenthesis.

hormone manipulation. All birds were between 0.59 and 1.77 years old with an average age of 1.2 years at the time of song exposure. All procedures were approved by the Institutional Animal Care and Use Committee of Michigan State University.

\section{2 | Hormone manipulation}

Three groups were created: circulating $E_{2}$ was (i) increased via implants and (ii) decreased using fadrozole injections; and (iii) control birds were treated with appropriate vehicles. Because fadrozole is water soluble and is commonly administered via injections in a saline vehicle, and $E_{2}$ is lipid soluble and most easily administered via longterm treatment in Silastic capsules, ${ }^{47}$ we gave each group both an implant and daily injections to ensure that all treatment groups experienced the same manipulations. In accordance with a previous study, ${ }^{48}$ $\mathrm{E}_{2}$ capsules were created by packing $2 \mathrm{~mm}$ of $17 \beta-\mathrm{E}_{2}$ (Steraloids, Newport, RI, USA) into a 5-mm length of Silastic tubing and sealing the ends with silicone. Blank pellets were sealed without packing with hormone. Birds were fully anaesthetised with isoflurane and a blank or $\mathrm{E}_{2}$ pellet was implanted s.c. over the left breast muscle, and the incision was sealed with collodion. All birds were then injected with $0.05 \mathrm{~mL}$ of $0.1 \mathrm{mg} \mathrm{mL}^{-1}$ Eloxiject (Henry Schein Animal Health, Dublin, $\mathrm{OH}, \mathrm{USA}$ ) i.p. for analgesia.

Birds implanted with $E_{2}$ pellets were injected with $10 \mu \mathrm{L}$ of saline into the right breast muscle. Those administered blank pellets were injected with $10 \mu \mathrm{L}$ of saline (control group) or $10 \mu \mathrm{L}$ of fadrozole (10 $\mu \mathrm{g} \mathrm{L} \mathrm{L}^{-1}$; Sigma-Aldrich, St Louis, MO, USA) (fadrozole group) into the right breast muscle. The initial injection was administered immediately following the implant surgery when birds were anaesthetised. Birds were then housed in small cages with one or two other birds of the same treatment group and sex, in a room where auditory and visual contact with the birds in the aviaries was maintained. On each of the following 6 days, each bird was given the same injection of saline or fadrozole into the right breast muscle as had been initially received. The effect of fadrozole on aromatase activity was not directly assessed in the present study, although previous research has indicated that this schedule and dose of fadrozole treatment results in a two-third reduction in aromatase activity in the telencephalon of adult male and female zebra finches 24 hours following the last injection. ${ }^{26}$

\section{3 | Stimulus exposure}

One day following the final injection of saline or fadrozole, birds were exposed to auditory stimuli to evaluate induced ZENK expression. Rhythmic and arrhythmic zebra finch songs were utilised from our previous studies on un-manipulated adult ${ }^{41}$ and juvenile (unpublished data) zebra finches. All songs used in this experiment were novel to all subjects. Rhythmic stimuli were natural zebra finch songs with no alteration to the timing of the syllables. Arrhythmic stimuli were created using the same songs utilised as rhythmic stimuli and modifying the duration of the inter syllable intervals aiming to disrupt the natural rhythmic structure of the songs. Nine songs of each type were generated, and three subsets of the songs each containing three songs were assembled. Birds were placed, one at a time, into an acoustic isolation chamber (252-Mini Sound Shelter; IAC Acoustics, Bronx, NY, USA), a novel environment, and allowed to habituate for 1 hour. Each bird was then exposed to one of the subsets of three songs, with all songs presented at approximately $70 \mathrm{~dB}$. One of the three songs was repeated for 30 seconds, followed by 30 seconds of silence. This pattern was repeated for 30 minutes, with the order of the songs being determined randomly. Immediately following the presentation of the stimuli, the birds were euthanised by rapid decapitation, and the brains were flash frozen in methyl butane to capture peak expression of ZENK mRNA. ${ }^{49}$ Blood was collected from the neck following decapitation for analysis 
of $\mathrm{E}_{2}$ levels. Blood samples were centrifuged for 10 minutes at $10621 \mathrm{~g}$ at $4^{\circ} \mathrm{C}$ and plasma was separated and stored at $-80^{\circ} \mathrm{C}$ until processed for radioimmunoassay. Retention of implants was confirmed following death.

All song exposures were recorded using a Vixia HF R300 camcorder (Canon USA, Melville, NY, USA). Recordings were reviewed to ensure birds did not sing and no extraneous background noise existed that might influence their auditory responses.

\section{4 | Radioimmunoassay}

To obtain a general estimate of effectiveness of the hormone manipulations, a single radioimmunoassay was conducted in a manner adapted from Svec and Wade. ${ }^{48}$ Parallelism and accurate detection of known quantities of $E_{2}$ were first demonstrated with recently collected zebra finch plasma (data not shown). Samples were analysed in three individuals from each combination of conditions (two sexes, three hormone manipulations and two types of auditory stimuli). To provide an estimate of recovery following extraction, $2000 \mathrm{dpm}$ of ${ }^{3} \mathrm{H}_{-E_{2}}$ (70 $\mathrm{Ci} \mathrm{mmol}^{-1}$; NET317250UC; Perkin Elmer, Waltham, MA, USA) was incubated at $4^{\circ} \mathrm{C}$ overnight with $100 \mu \mathrm{L}$ of plasma from control and fadrozole treated birds and $40 \mu \mathrm{L}$ of plasma from $\mathrm{E}_{2}$ treated birds. Steroids were then extracted twice with diethyl ether and then samples were dried under nitrogen. They were resuspended in $5 \mu \mathrm{L}$ of $100 \%$ ethanol and then combined with $500 \mu \mathrm{L}$ of phosphate-buffered saline (PBS) with gelatin and stored overnight at $4^{\circ} \mathrm{C}$. A competitive binding assay was completed in duplicate samples, with a serially diluted standard curve $\left(0.98-250 \mathrm{pg} \mathrm{E}_{2}\right)$ in triplicate, by adding an $\mathrm{E}_{2}$ antibody (7010-2650; Bio-Rad AbD Serotec Inc., Raleigh, NC, USA) with ${ }^{3} \mathrm{H}-\mathrm{E}_{2}$ and incubating overnight at $4^{\circ} \mathrm{C}$. Six aliquots of a sample containing a known concentration of $\mathrm{E}_{2}$ were used to determine intraassay precision, and water blanks were added as controls $(n=4)$. The next day, dextran-coated charcoal $(0.025 \%$ dextran and $0.25 \%$ charcoal in PBS) was added and centrifuged at $1934 \mathrm{~g}$ for 25 minutes at $4^{\circ} \mathrm{C}$ to remove unbound tracer. The remaining sample was combined with scintillation fluid (UltimaGold; Perkin Elmer) and analysed with a scintillation counter (Tri-Carb 2910 TR; Perkin Elmer). $E_{2}$ levels were calculated by standardising samples for individual recovery (ranging from $50 \%$ to $82 \%$ ) and the volume was assayed and compared with the standard curve. The intra-assay coefficient of variation was $12.7 \%$.

\section{5 | Tissue processing}

Brains were sectioned coronally into six series at $20 \mu \mathrm{m}$ and thaw mounted onto SuperFrost Plus slides (Fisher Scientific, Hampton, $\mathrm{NH}$, USA). All series were stored at $-80^{\circ} \mathrm{C}$ until further processing. One series from each animal was stained with thionin to facilitate identification of neuroanatomy.

ZENK mRNA expression in individual cells was visualised by in situ hybridisation. Bacteria containing a pBlueScript SK+ plasmid with a clone of the zebra finch zenk gene were obtained from the Songbird ESTIMA clone collection. ${ }^{50}$ Bacteria were grown overnight on lysogeny broth (LB) agar plates containing $100 \mu \mathrm{g} \mathrm{mL}^{-1}$ ampicillin.
Individual colonies were selected and allowed to grow overnight in LB with $100 \mu \mathrm{g} \mathrm{mL}^{-1}$ ampicillin. DNA was isolated using the Wizard Plus SV Minipreps DNA Purification System (Promega, Madison, WI, USA) and the sequence of the insert was confirmed in both directions. Bacteria were then regrown in LB with $100 \mu \mathrm{g} \mathrm{mL}^{-1}$ ampicillin and DNA was isolated using the NucleoBond Xtra Maxi kit (Macherey-Nagel, Bethlehem, PA, USA). The DNA was linearised with Sall (T3) and EcoRI (T7) restriction enzymes and stored at $-20^{\circ} \mathrm{C}$. Antisense (T3) and sense (T7) probes were transcribed in accordance with the manufacturer instructions for the Digoxigenin RNA Labeling Kit (Roche Diagnostics, Indianapolis, IN, USA). The probes were purified by filtering through a column made with $\mathrm{G} 50$ Sephadex beads and stored at $-80^{\circ} \mathrm{C}$ overnight.

As a result of the large number of brain samples, tissue was processed in four runs with all groups represented in every run. One series of slides from each animal was processed using in situ hybridisation for ZENK with antisense probes, and a second series from a minimum of two animals was used in each run for a control to confirm the absence of labelling with sense probes (not shown). Tissue was warmed to room temperature and then fixed in $4 \%$ paraformaldehyde for 10 minutes. Slides were rinsed $3 \times 3$ minutes in $0.1 \mathrm{~mol} \mathrm{~L}^{-1} \mathrm{PBS}$, and incubated in $0.25 \%$ acetic anhydride in triethanolamine-hydrochloride for 10 minutes. They were then washed $3 \times 5$ minutes in PBS and allowed to equilibrate in hybridisation buffer for 1 hour. Slides were incubated at $56^{\circ} \mathrm{C}$ overnight in

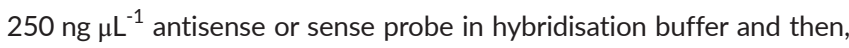
the next day, they were rinsed $2 \times 5$ min in $2 \times$ saline-sodium citrate (SSC) at $60^{\circ} \mathrm{C}$. The slides were then incubated in $20 \mu \mathrm{g} \mathrm{m}^{-1} \mathrm{RNase}$ in $2 \times \mathrm{SSC}$ at $37^{\circ} \mathrm{C}$ for 30 minutes, rinsed for 15 minutes in $0.2 \times \mathrm{SSC}$ at $37^{\circ} \mathrm{C}$, and then $3 \times 5$ minutes in $0.2 \times S S C$ at $60^{\circ} \mathrm{C}$. The brain sections were allowed to return to room temperature, then rinsed $3 \times 5$ minutes in maleic acid buffer with $0.1 \%$ Tween-20 (MABT). The slides were incubated in $0.9 \%$ $\mathrm{H}_{2} \mathrm{O}_{2}$ in MABT for 30 minutes and rinsed $3 \times 5$ minutes in MABT. They were incubated in $5 \%$ normal sheep serum in MABT for 30 minutes, then rinsed $3 \times 5$ minutes in MABT. Next, the slides were incubated in $0.5 \mu \mathrm{L} \mathrm{mL}^{-1}$ anti-digoxigenin-AP Fab fragments (Roche Diagnostics) in MABT for 2 hours, and then rinsed $2 \times 3$ minutes in MABT. Slides were rinsed $3 \times 5$ minutes in detection buffer to equilibrate. The colour reaction was performed by incubating slides in $4.5 \mu \mathrm{L} \mathrm{mL}^{-1} \mathrm{NBT}$ and $3.5 \mu \mathrm{L} \mathrm{mL}{ }^{-1}$ BCIP (Roche Diagnostics) in detection buffer for 1 hour and 55 minutes. This produced a blue reaction product in specific cells in sections treated with the antisense probe and no labelling in those exposed to the sense probe. Slides were rinsed $3 \times 5$ minutes in TE buffer to stop the colour reaction, and then dehydrated and coverslipped with VectaMount (Vector Laboratories Inc., Burlingame, CA, USA). A qualitative assessment of the overall staining revealed numerous brain regions with minimal or no ZENK labelling, including HVC and the robust nucleus of the arcopallium, indicating the specificity of the response in auditory and social behaviour network regions.

An investigator who was blind to the stimulus exposure condition, sex and age of the birds conducted analysis of all slides using IMAGEJ (National Institutes of Health, Bethesda MD, USA). All regions of interest were analysed bilaterally in two adjacent sections. Boxes were placed within the NCM, CMM and Tn as described and depicted previously, ${ }^{41}$ and all cells containing a blue filled cytoplasm 
and a clear hole of a nucleus that were the appropriate size and shape were counted. For NCM, a $0.525 \times 0.393 \mathrm{~mm}$ box was placed with the medial corner under the hippocampus at the point where the ventricle begins to curve ventrally to run parallel with the midline. For CMM, a $0.496 \times 0.205 \mathrm{~mm}$ box was placed the ventricle just lateral to where it curves ventrally toward the midline between A 1.6 and A 1.2 from a songbird brain atlas. ${ }^{51}$ For Tn, a $0.238 \times 0.244 \mathrm{~mm}$ box was placed near the ventral edge of the telencephalic lobe where a corner is formed by the ventral and medal edges of the lobe. The density of ZENK expressing cells (labelled nuclei per unit area) was calculated for each brain region and average density values were calculated for each region of interest in each hemisphere.

\section{6 | Statistical analysis}

A few animals were excluded from analysis of a particular brain region as a result of damage to individual tissue sections. Final sample sizes are indicated in Table 1. Separate mixed model ANOVAs were conducted for each brain region with a hemisphere as a within subjects factor and sex, hormone manipulation and stimulus type (rhythmic or arrhythmic) as between subjects factors. A main effect of hormone in the CMM was followed with a post-hoc Scheffe test to determine which pairs of hormone conditions differed. Two-way and three-way interactions detected within the NCM were each probed with subsequent ANOVAs within groups. Bonferroni corrections were used to adjust for multiple comparisons, with adjusted $\alpha$-levels being indicated with each result. A one-way ANOVA was conducted on plasma oestradiol levels with hormone manipulation as a factor. Because only a subset of the birds in each group was evaluated, the data were collapsed across sex and stimulus type; we assumed that these factors would not influence estimates of the effectiveness of $\mathrm{E}_{2}$ treatments. All statistics were calculated using sPSS (IBM Corp., Armonk, NY, USA).

\section{RESULTS}

\section{1 | Hormone manipulation}

Two samples were eliminated from analysis as a result of technical mistakes. Five samples, two from fadrozole-treated and three from the control birds, were below the limit of detectability for this assay. To be conservative, they were assigned the lowest detectable value for the purpose of statistical analysis. A significant effect of hormone treatment on circulating $E_{2}$ levels was detected $\left(F_{2,31}=150.10, P<.001\right)$ (Figure 1). A post-hoc Scheffe test indicated a greater concentration of the hormone in the $\mathrm{E}_{2}$-treated compared to both the control and fadrozoletreated groups (both $P<.05$ ). However, the values were not significantly different in the control and fadrozole-treated groups $(P>.05)$.

\section{2 | Caudomedial mesopallium}

A significant main effect of hemisphere was detected, with a greater density of ZENK expressing cells in the left hemisphere than the right $\left(F_{1,88}=35.917, P<.001\right)$ (Figure 2). A main effect of sex was detected $\left(F_{1,88}=8.557, P=.004\right)$ (Figure 2$)$, such that females had a greater density of ZENK expressing cells than males. The hormone manipulation also produced a significant effect on ZENK expression $\left(F_{2,88}=3.468, P=.036\right)$ (Figure 2). Although post-hoc comparisons indicated no significant differences between any of the three groups, on average, the density of ZENK+ cells was highest in the control birds compared to those with hormone manipulations. No main effect of rhythm stimulus type was detected $\left(F_{1,88}=1.308, P=.256\right)$ and no significant interactions were detected between any of the variables (all $F<1.338, P>$.267). Data for individual groups are presented in Table 1.

\section{3 | Caudomedial nidopallium}

As seen in the CMM, a significant main effect of hemisphere was detected with a greater density of ZENK expressing cells on the left compared to the right $\left(F_{1,88}=8.586, P=.004\right)$ (Figure 3). An increase in these cells was also found in males compared to females $\left(F_{1,88}=4.253, P=.042\right)$ (Figure 3). No main effects of stimulus rhythmicity $\left(F_{1,88}=0.014, P=.908\right)$ or hormone manipulation $\left(F_{2,88}=0.886\right.$, $P=.416)$ were detected.

Two interactions were detected among variables, which are described immediately below. None of the other possible interactions among hemisphere, sex, rhythm type and treatment were statistically significant (all $F<2.578, P>$.081). Data for the individual groups are presented in Table 1.

First, there was an interaction between sex and hormone treatment $\left(F_{2,88}=4.196, P=.018\right)$ (Figure 4). This result was probed in two ways. We conducted separate one-way ANOVAs within each sex with hormone condition as a factor and no effect of hormone treatment was detected in males or females $\left(F_{2,43}=2.915, P=.065 ; F_{2,51}=1.541\right.$, $P=.224$, respectively; $\alpha=0.025$ ). We also used independent $t$-tests

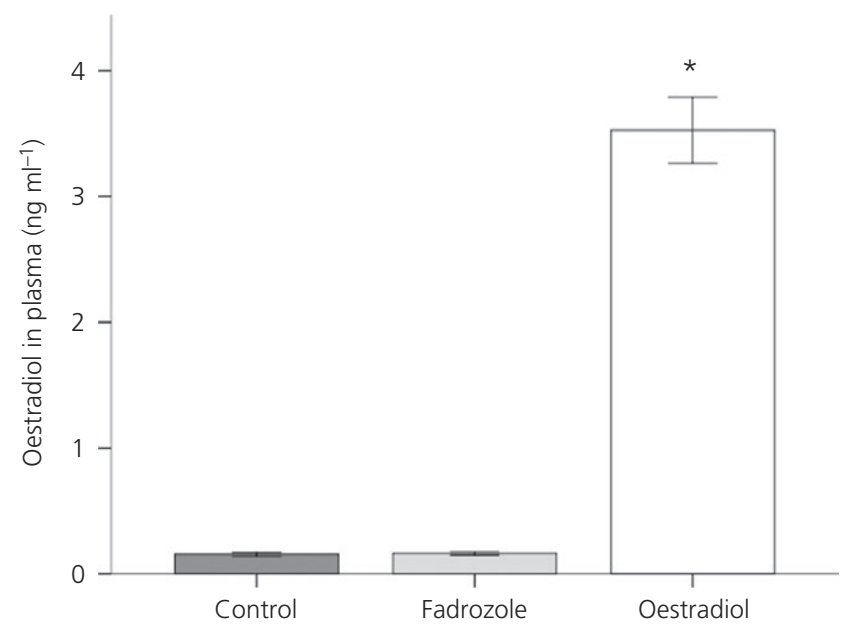

FIGURE 1 Concentration of oestradiol detected in plasma. A significant increase in the concentration detected in oestradioltreated compared to control or fadrozole-treated birds is indicated by an asterisk (*). No difference was detected between control and fadrozole treated birds 


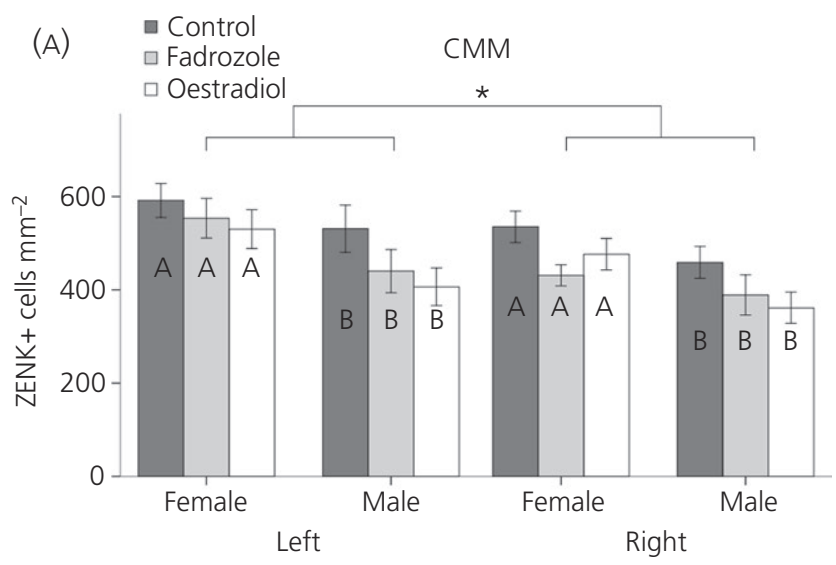

(B)

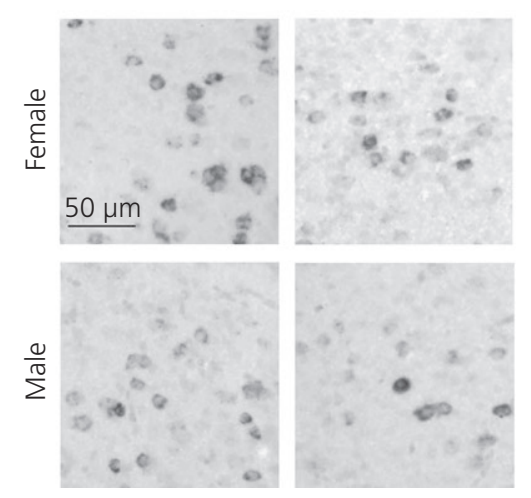

FIGURE 2 Density of cells expressing ZENK in the caudomedial mesopallium (CMM). (A) Depiction of this measure across sexes and hormone conditions (mean \pm SEM). Data are collapsed across stimulus type (rhythmic and arrhythmic) because no significant effects of this variable were detected. A main effect of hemisphere, with greater density of ZENK expression on the left, is indicated by an asterisk (*). A main effect of sex (female>male) is indicated by different uppercase letters within the bars. (B) Representative examples of ZENK expression in birds exposed to arrhythmic song in the left and right hemisphere of a female, and the left and right hemisphere of a male

to analyse the effect of sex within each of the hormone manipulations. Here, within control birds, a greater density of ZENK expressing cells was present in males compared to females $\left(t_{30}=3.728, P=.001\right.$; $\alpha=0.017$ ), whereas no effect of sex was detected in the fadrozole$\left(t_{30}=0.462, P=.648 ; \alpha=0.017\right)$ or $E_{2}$-treated $\left(t_{30}=0.465, P=.645\right.$; $\alpha=0.017$ ) animals.

Second, there was a significant three-way interaction between hemisphere, sex and rhythm stimulus type $\left(F_{1,88}=5.164, P=.025\right)$ (Figure 3). This result was further investigated with two-way, mixedmodel ANOVAs (hemispherexsex) within each auditory stimulus. A sex $x$ hemisphere interaction was detected in birds exposed to arrhythmic $\left(F_{1,48}=5.804, P=.020\right)$ but not rhythmic $\left(F_{1,48}=0.475, P=.494\right)$ song. Therefore, within birds exposed to the arrhythmic song only, paired $t$ tests were used to assess the effects of hemisphere within each of the two sexes. The only significant effect detected was an increased density of cells expressing ZENK mRNA in the left compared to the right hemisphere of arrhythmic song exposed males $\left(t_{22}=2.917, P=.008\right.$, $\alpha=0.0125$ ) (Figure 3).

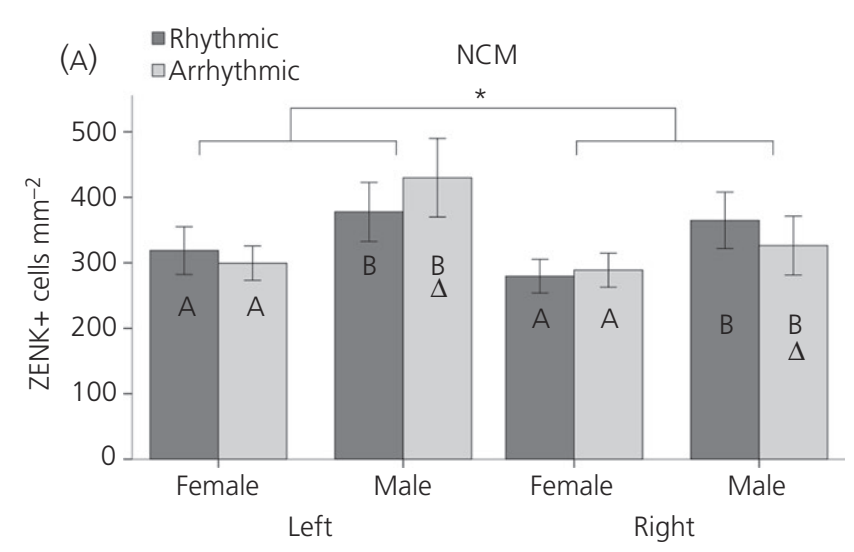

(B)

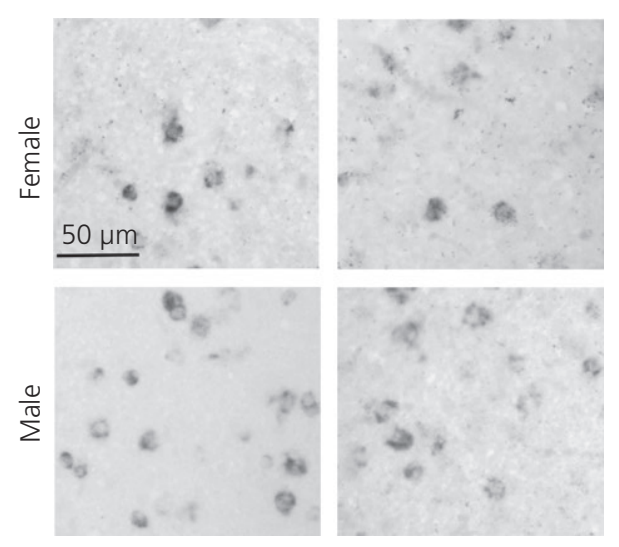

FIGURE 3 ZENK expressing cells in the caudomedial nidopallium (NCM). (A) Depiction of the density of cells containing ZENK mRNA across the sexes and auditory stimuli (mean \pm SEM). A main effect of hemisphere, with greater expression on the left, is indicated by an asterisk $(*)$. Different uppercase letters within the bars indicate a main effect of sex, with greater ZENK expression in males compared to females. A three-way interaction was detected. Greater ZENK density was seen on the left than the right in arrhythmic song exposed males, indicated by a triangle $(\Delta)$. (B) Representative samples of ZENK expression in control males and females exposed to rhythmic song

\subsection{Nucleus taeniae}

Similar to both auditory regions, there was a significant main effect of hemisphere, with a greater density of ZENK expressing cells in the left compared to the right hemisphere $\left(F_{1,88}=18.936, P<.001\right)$. There were no main effects of sex $\left(F_{1,88}=2.545, P=.114\right)$, rhythm type $\left(F_{1,88}=2.324, P=.131\right)$ or hormone manipulation $\left(F_{2,88}=1.963, P=.147\right)$. There were also no significant interactions between any combination of hemisphere, sex, rhythm type and hormone (all $F<2.606, P>.079$ ). Data are presented in Table 1.

\section{4 | DISCUSSION}

\subsection{Summary of specific effects}

Across the NCM, CMM and Tn, a greater density of cells expressing ZENK mRNA was detected in the left hemisphere. Sex differences in ZENK expression were detected in both auditory regions, although in opposite directions, with a higher density of cells in males in the 


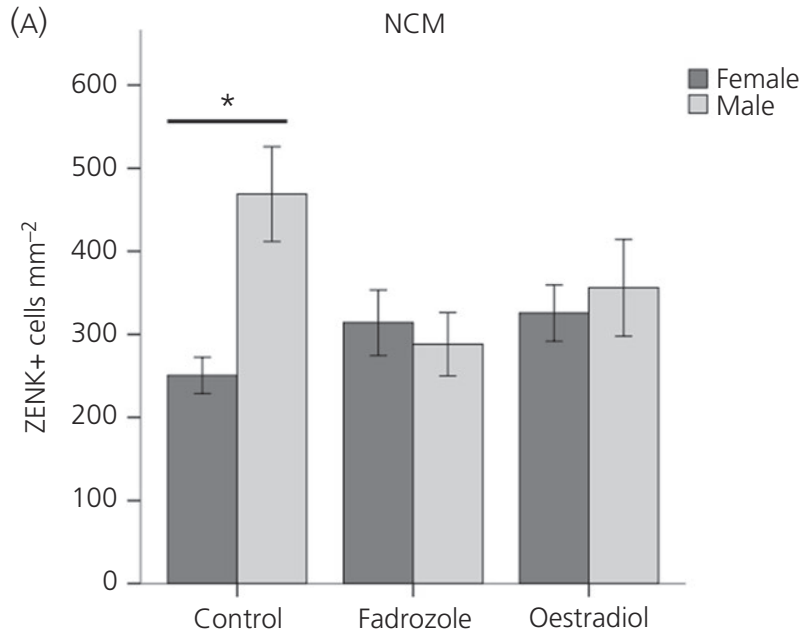

(B)

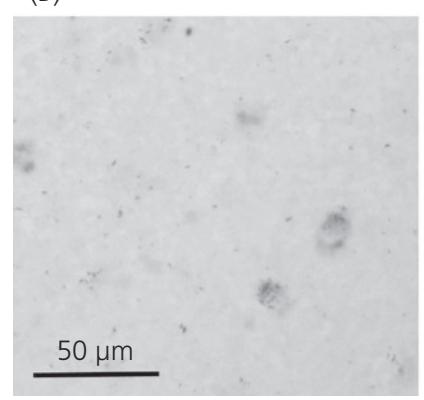

Female

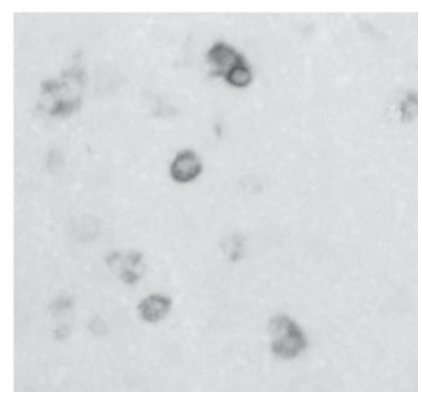

Male
FIGURE 4 ZENK expression in the the caudomedial nidopallium (NCM) of males and females across hormone manipulations. (A) Depiction of the density of cells (mean \pm SEM) across the three treatment groups. An interaction was detected; expression was greater in males than females only in the control animals, indicated by an asterisk $\left({ }^{*}\right)$. (B) Representative images from a control female and male

NCM and in females in the CMM. An effect of hormone manipulation was also seen in the CMM, and, although no pairwise comparisons were statistically significant, the pattern suggested greater ZENK expression in the control birds compared to both the $E_{2}$ - and fadrozoletreated groups, across both sexes and song stimuli.

Statistically significant interactions were detected just in the NCM. In the control group only, the density of cells expressing ZENK mRNA was greater in males than females. ZENK expressing cells were also increased in the left compared to the right hemisphere in males exposed to arrhythmic but not rhythmic, song. Thus, these groups contributed substantially to the main effect of hemisphere. In the present study, the effects of rhythmicity of the song stimulus were limited, although they may have been reduced by particular methodological differences from our earlier studies. ${ }^{41}$

\section{2 | Methodological issues}

The lack of effects as a result of song stimulus type differs from our previous finding of an increased density of ZENK+ cells in response to arrhythmic compared to rhythmic song across all three brain areas investigated. ${ }^{41}$ Several methodological aspects may relate to the differences between studies. For example, birds in the present study were housed with only one or two conspecifics, whereas they were kept in large group aviaries in our earlier work. It is possible that the current conditions were more stressful. Keeping zebra finches individually housed at the same time as maintaining auditory and visual contact with conspecifics can cause changes in ZENK expression in the social behaviour network, which includes $\mathrm{Tn}^{52}$ The experience of undergoing anaesthesia, a hormone implant, and a week of daily injections likely also induced some stress compared to our earlier study on adults. Future studies could assess cortisol levels to assess the stress effects of the manipulations.

Perhaps the most important difference between our studies, however, involved the quantification of ZENK protein in the earlier work ${ }^{41}$ versus mRNA in the present study. This change was made because the antibody used in the previous study was no longer available. The relationship between mRNA and protein expression can vary widely between genes and tissue types. ${ }^{53,54}$

Previous studies assessing the timeline of peak ZENK mRNA expression in the zebra finch brain have not compared across sexes, hemispheres or brain regions. Thus, without an unstimulated control group for comparison, the possibility remains that sex and laterality differences detected in the present study were influenced by auditory exposure prior to our testing, as a result of possible variability in the ZENK expression timeline.

The results of the present study also differ from studies indicating the capacity of $E_{2}$ to improve auditory discrimination to types of stimuli not used in the present experiment, such as conspecific vs heterospecific song, tones, reverse song or white noise. ${ }^{23,24,27,28,55}$ It is unclear whether $E_{2}$ can also modulate responses to auditory rhythms because the anticipated difference between our stimulus types was not detected. Further work is needed to resolve this issue. NCM is a large, heterogeneous brain region. In white-throated sparrows, $E_{2}$ enhances the ZENK response to song compared to tones in the rostral and medial portions of NCM but not in other regions of the nucleus. ${ }^{55}$ The white-throated sparrow brains were sectioned in the sagittal plane, ${ }^{55}$ whereas the brains in the present study were sectioned coronally to maintain consistency with our earlier work. ${ }^{41}$ Although the area of NCM analysed here is likely in the rostral region described in the sparrow study, it is difficult to make exact comparisons across different planes of section. It is possible that regions other than where we sampled might show an effect of $E_{2}$.

It is also possible that the hormone manipulations did not provide sufficiently large differences in brain $E_{2}$ availability from the control group, although we think this is unlikely. Although our assay was not sufficiently sensitive to detect a significant reduction in circulating $E_{2}$ in fadrozole compared to the control birds, the same fadrozole administration over only 6 days substantially reduces aromatase in the forebrain as a whole, ${ }^{26}$ where NCM, CMM and Tn are located. The pattern of ZENK expression across manipulations also indicates that fadrozole was effective in the brain because the sex difference detected in the NCM in control animals was eliminated by this drug. 
A final methodological consideration is the duration of the hormone manipulations. $E_{2}$ is rapidly synthesised in the NCM of zebra finches in response to conspecific song. ${ }^{22,23}$ In addition, the effects of $E_{2}$ on auditory responses can appear rapidly and are likely mediated through a non-classical membrane bound receptor such as GPER1 (previously known as GPR30). ${ }^{14}$ Continuous exposure to $E_{2}$ for 1 week may have overwhelmed these rapid $E_{2}$ responses, washing out any modulation of auditory response that increased $E_{2}$ may facilitate. We chose to use a longer-term $E_{2}$ modulation because it provided a minimally invasive way to influence $E_{2}$ levels that had the possibility of impacting modulation through multiple $E_{2}$ receptor types. ${ }^{26}$ Future studies using infusion of fadrozole or $E_{2}$ into the NCM could allow for an assessment of the rapid effects of $E_{2}$ on rhythm perception.

\section{3 | Lateralisation}

The finding in the present study of a higher density of cells expressing ZENK mRNA in the left hemisphere across brain regions adds to a complex literature on lateralisation of auditory perception. In humans, left lateralisation of linguistic functions appears consistent across production and perception (29). These results parallel the left lateralisation following perception of conspecific vocalisations in the present study. However, the literature available for songbirds is less consistent than that available for humans. Similar to the present experiment, increased ZENK expression was detected in the left NCM of juvenile zebra finches exposed to novel conspecific song. ${ }^{30}$ However, the same study did not find lateral differences in adults. Our previous work on juvenile zebra finches, using the same auditory stimuli as the present study, did not initially assess hemisphere as a factor (unpublished data), although further analysis of the data reveals no significant differences in the density of cells expressing ZENK protein between the left and right sides of the brain. Electrophysiological studies have also provided mixed results. Increased response strength in the left hemisphere has been detected among fast-learning birds trained in a GO/NoGO paradigm. ${ }^{31}$ Zebra finches exposed to 4 or 9 days of a heterospecific acoustic environment also showed left lateralisation of electrophysiological responses in the NCM, ${ }^{32}$ whereas those housed under typical conditions with conspecific song exposure showed right lateralisation of NCM activity. ${ }^{32,33}$ A functional magnetic resonance imaging (fMRI) study indicated greater differences in response strength between bird's own song, novel conspecific song, tutor song and tones in the right hemisphere of the zebra finch brain. ${ }^{34}$ By contrast, in another fMRI study, the data suggest greater activation of the left hemisphere (although lateralisation was not specifically assessed). ${ }^{56}$ The same study also found greater influence of aromatase inhibition on neural activity in the left hemisphere. ${ }^{56}$ Given the inconsistencies that can be detected across different measures of neural activity, and the fact that it is possible for neurones to fire without inducing ZENK expression, ${ }^{57}$ further work is necessary to draw conclusions about the functional significance of lateral differences in gene expression detected in the present study.

\section{4 | Caudomedial mesopallium}

The sex difference in the CMM, with greater ZENK expression in females compared to males, parallels results from our previous study with adult zebra finches without hormone manipulations. ${ }^{41}$ The quality of songs is an indicator to females of the fitness of males. ${ }^{58}$ Thus, the consistent increase in activity in females compared to males in the CMM may relate to female use of the CMM for evaluation of songs of potential mates, a function typically not employed by males.

The trend for increased ZENK expression in control animals compared to those treated with either $E_{2}$ or fadrozole suggests that an optimal level of the hormone may exist. This pattern of results is consistent with a study that found a trend in the NCM for a reduced electrophysiological response to novel conspecific song in fadrozoleand $E_{2}$-treated birds compared to saline-treated controls. ${ }^{59}$ Taken together, these results suggest that too much or too little available $E_{2}$ within the auditory forebrain may inhibit optimal neural responses to auditory stimuli. Fadrozole can reduce both the aromatase activity within the zebra finch brain ${ }^{26}$ and the total amount of $E_{2}$ present in the telencephalon. ${ }^{60}$ Although our assay did not detect differences in plasma $E_{2}$ levels between the control and fadrozole treated birds, those receiving the oestrogen synthesis inhibitor likely experienced less $E_{2}$ availability in the auditory brain as a result of decreased local synthesis, as well as a possible reduction in the $\mathrm{E}_{2}$ released within the NCM during song exposure. ${ }^{22,23}$

\subsection{Caudomedial nidopallium}

By contrast to the effect seen in the CMM, a greater density of cells expressing ZENK mRNA was seen in males compared to females in the NCM. Although this effect was not detected in our previous study, ${ }^{41}$ a similar pattern of activity was detected in the NCM of zebra finches using electrophysiology, with males showing an enhanced response to novel conspecific songs compared to females. ${ }^{59}$ The interaction between hormone manipulation and sex in the present study indicates that this sex difference is limited to the control animals. Similar to the present data from the CMM, this result suggests that there may be an optimal level of circulating $E_{2}$, and an increase or decrease can diminish sex differences in auditory processing. In addition, the opposite directions of sex differences between the NCM and CMM may indicate that these areas are functionally specialised. Specifically, the NCM may be more involved in processing by males for nest site defence, whereas the CMM may aid in processing of song for value as a potential mate.

The interaction between sex, hemisphere and rhythmicity of the song stimuli in the NCM could present a challenge for interpretation. A significant increase in ZENK expression in the left relative to the right hemisphere was detected only in males exposed to arrhythmic song; it is difficult to speculate on potential reasons for such a specific effect. However, a trend for a greater density of ZENK+ cells in the left hemisphere existed in all other combinations of sex and rhythm type. Thus, although data from these males was largely responsible for the 
main effect of lateralisation in NCM, it is unlikely that this interaction represents a functional difference across groups.

\subsection{Nucleus taeniae}

The lack of effect of stimulus rhythmicity detected in Tn contrasts with the results previously seen in adults that did not receive hormone manipulations, ${ }^{41}$ although both studies are consistent in finding no sex differences in ZENK expression in Tn. It would be parsimonious to suggest that the hormone manipulations in the present study eliminated the increased response to arrhythmic song; ${ }^{41}$ however, the control birds also responded similarly to the two types of auditory stimuli (Table 1). Thus, other differences from our previous study likely influenced the results.

\section{5 | CONCLUSIONS}

Overall, the results of the present study suggest a strong left lateralisation of neural activity in response to conspecific songs across brain regions, regardless of $E_{2}$ levels or rhythm type. This effect parallels the lateralisation of language seen in humans. In addition, the data suggest the possibility of an optimal level of $E_{2}$ at which these neural responses are strongest. Sex differences were also opposite between auditory regions, with greater activity in the NCM in males and in the CMM in females. Further studies are needed to clarify potential relationships between $E_{2}$ and rhythm perception, as well as the functional significance of sex differences and lateral differences in gene expression.

\section{ACKNOWLEDGEMENTS}

We thank Kelsey Stevenson and Rachel Smeenge for analysis of the videos and technical assistance; Katherine Jones for creation of the song stimuli; Camilla Peabody for conducting the radioimmunoassay; and Claudio Mello for providing the ZENK clone. This work was partially funded by National Institutes of Health R01-MH096705 and Michigan State University's program for Research in Autism, Intellectual and Neurodevelopmental Disabilities (RAIND).

\section{CONFLICT OF INTERESTS}

The authors declare that they have no conflicts of interest.

\section{ORCID}

J. Lampen (iD http://orcid.org/0000-0001-6825-6828

\section{REFERENCES}

1. Wickham LA, Gao J, Toda I, Rocha EM, Ono M, Sullivan DA Identification of androgen, estrogen and progesterone receptor mRNAs in the eye. Acta Ophthalmol Scand. 2000;78:146-153.
2. Kobayashi K, Kobayashi H, Ueda M, Honda Y. Estrogen receptor expression in bovine and rat retinas. Invest Ophthalmol Vis Sci. 1998;39:2105-2110.

3. Begay V, Valotaire Y, Ravault JP, Collin JP, Falcon J. Detection of estrogen receptor mRNA in trout pineal and retina: estradiol-17 beta modulates melatonin production by cultured pineal photoreceptor cells. Gen Comp Endocrinol. 1994;93:61-69.

4. Barni T, Maggi M, Fantoni G, et al. Sex steroids and odorants modulate gonadotropin-releasing hormone secretion in primary cultures of human olfactory cells. J Clin Endocrinol Metab. 1999;84: 4266-4273.

5. Stenberg AE, Wang H, Sahlin L, Hultcrantz M. Mapping of estrogen receptors alpha and beta in the inner ear of mouse and rat. Hear Res. 1999;136:29-34.

6. Noirot IC, Adler HJ, Cornil CA, et al. Presence of aromatase and estrogen receptor alpha in the inner ear of zebra finches. Hear Res. 2009;252:49-55.

7. Maruska KP, Fernald RD. Steroid receptor expression in the fish inner ear varies with sex, social status, and reproductive state. BMC Neurosci. 2010;11:58.

8. Walpurger V, Pietrowsky R, Kirschbaum C, Wolf OT. Effects of the menstrual cycle on auditory event-related potentials. Horm Behav. 2004:46:600-606.

9. Tillman GD. Estradiol levels during the menstrual cycle differentially affect latencies to right and left hemispheres during dichotic listening: an ERP study. Psychoneuroendocrinology. 2010;35:249-261.

10. Coleman JR, Campbell D, Cooper WA, Welsh MG, Moyer J. Auditory brainstem responses after ovariectomy and estrogen replacement in rat. Hear Res. 1994;80:209-215.

11. Golub MS, Germann SL, Hogrefe CE. Endocrine disruption and cognitive function in adolescent female rhesus monkeys. Neurotoxicol Teratol. 2004;26:799-809.

12. Caruso S, Maiolino L, Rugolo S, et al. Auditory brainstem response in premenopausal women taking oral contraceptives. Hum Reprod. 2003;18:85-89

13. Kuenzel WJ, Medina L, Csillag A, Perkel DJ, Reiner A. The avian subpallium: new insights into structural and functional subdivisions occupying the lateral subpallial wall and their embryological origins. Brain Res. 2011;1424:67-101.

14. Remage-Healey L, Jeon SD, Joshi NR. Recent evidence for rapid synthesis and action of oestrogens during auditory processing in a songbird. J Neuroendocrinol. 2013;25:1024-1031.

15. Heimovics SA, Prior NH, Maddison CJ, Soma KK. Rapid and widespread effects of 17beta-estradiol on intracellular signaling in the male songbird brain: a seasonal comparison. Endocrinology. 2012;153: 1364-1376.

16. Shen P, Campagnoni CW, Kampf K, Schlinger BA, Arnold AP, Campagnoni AT. Isolation and characterization of a zebra finch aromatase CDNA: in situ hybridization reveals high aromatase expression in brain. Brain Res Mol Brain Res. 1994;24:227-237.

17. Balthazart J, Absil P, Foidart A, Houbart M, Harada N, Ball GF. Distribution of aromatase-immunoreactive cells in the forebrain of zebra finches (Taeniopygia guttata): implications for the neural action of steroids and nuclear definition in the avian hypothalamus. $J$ Neurobiol. 1996;31:129-148.

18. Saldanha CJ, Tuerk MJ, Kim YH, Fernandes AO, Arnold AP, Schlinger $B A$. Distribution and regulation of telencephalic aromatase expression in the zebra finch revealed with a specific antibody. J Comp Neurol. 2000;423:619-630.

19. Gahr M, Flugge G, Guttinger HR. Immunocytochemical localization of estrogen-binding neurons in the songbird brain. Brain Res. 1987;402:173-177.

20. Jacobs EC, Arnold AP, Campagnoni AT. Zebra finch estrogen receptor cDNA: cloning and mRNA expression. J Steroid Biochem Mol Biol. 1996;59:135-145. 
21. Acharya KD, Veney SL. Characterization of the G-protein-coupled membrane-bound estrogen receptor GPR30 in the zebra finch brain reveals a sex difference in gene and protein expression. Dev Neurobiol. 2012;72:1433-1446.

22. Remage-Healey L, Maidment NT, Schlinger BA. Forebrain steroid levels fluctuate rapidly during social interactions. Nat Neurosci. 2008;11:1327-1334.

23. Remage-Healey L, Dong SM, Chao A, Schlinger BA. Sex-specific, rapid neuroestrogen fluctuations and neurophysiological actions in the songbird auditory forebrain. J Neurophysiol. 2012;107:1621-1631.

24. Remage-Healey L, Coleman MJ, Oyama RK, Schlinger BA. Brain estrogens rapidly strengthen auditory encoding and guide song preference in a songbird. Proc Natl Acad Sci USA. 2010;107:3852-3857.

25. Remage-Healey L, Joshi NR. Changing neuroestrogens within the auditory forebrain rapidly transform stimulus selectivity in a downstream sensorimotor nucleus. J Neurosci. 2012;32:8231-8241.

26. Wade J, Schlinger BA, Hodges L, Arnold AP. Fadrozole: a potent and specific inhibitor of aromatase in the zebra finch brain. Gen Comp Endocrinol. 1994;94:53-61.

27. Maney DL, Cho E, Goode CT. Estrogen-dependent selectivity of genomic responses to birdsong. Eur J Neurosci. 2006;23:1523-1529.

28. Maney DL, Goode CT, Lange HS, Sanford SE, Solomon BL. Estradiol modulates neural responses to song in a seasonal songbird. J Comp Neurol. 2008;511:173-186.

29. Doupe AJ, Kuhl PK. Birdsong and human speech: common themes and mechanisms. Annu Rev Neurosci. 1999;22:567-631.

30. Moorman S, Gobes SM, Kuijpers M, Kerkhofs A, Zandbergen MA, Bolhuis JJ. Human-like brain hemispheric dominance in birdsong learning. Proc Natl Acad Sci USA. 2012;109:12782-12787.

31. Bell BA, Phan ML, Vicario DS. Neural responses in songbird forebrain reflect learning rates, acquired salience, and stimulus novelty after auditory discrimination training. J Neurophysiol. 2015;113:1480-1492.

32. Yang LM, Vicario DS. Exposure to a novel stimulus environment alters patterns of lateralization in avian auditory cortex. Neuroscience. 2015;285:107-118.

33. Phan ML, Vicario DS. Hemispheric differences in processing of vocalizations depend on early experience. Proc Natl Acad Sci USA. 2010;107:2301-2306.

34. Voss HU, Tabelow K, Polzehl J, et al. Functional MRI of the zebra finch brain during song stimulation suggests a lateralized response topography. Proc Natl Acad Sci USA. 2007;104:10667-10672.

35. Grube M, Cooper FE, Griffiths TD. Auditory temporal-regularity processing correlates with language and literacy skill in early adulthood. Cogn Neurosci. 2013;4:225-230.

36. Muneaux M, Ziegler JC, Truc C, Thomson J, Goswami U. Deficits in beat perception and dyslexia: evidence from French. NeuroReport. 2004;15:1255-1259.

37. Wieland EA, McAuley JD, Dilley LC, Chang SE. Evidence for a rhythm perception deficit in children who stutter. Brain Lang. 2015;144:26-34.

38. Corriveau K, Pasquini E, Goswami U. Basic auditory processing skills and specific language impairment: a new look at an old hypothesis. $J$ Speech Lang Hear Res. 2007;50:647-666.

39. Corriveau KH, Goswami U. Rhythmic motor entrainment in children with speech and language impairments: tapping to the beat. Cortex. 2009;45:119-130.

40. Norton P, Scharff C. "Bird Song Metronomics": isochronous organization of zebra finch song rhythm. Front Neurosci. 2016;10:309.

41. Lampen J, Jones K, McAuley JD, Chang SE, Wade J. Arrhythmic song exposure increases ZENK expression in auditory cortical areas and nucleus taeniae of the adult zebra Finch. PLoS ONE. 2014;9:e108841.
42. Guenther FH, Ghosh SS, Tourville JA. Neural modeling and imaging of the cortical interactions underlying syllable production. Brain Lang. 2006;96:280-301.

43. Tourville JA, Reilly KJ, Guenther FH. Neural mechanisms underlying auditory feedback control of speech. Neurolmage. 2008;39:1429-1443.

44. Geiser E, Notter M, Gabrieli JD. A corticostriatal neural system enhances auditory perception through temporal context processing. J Neurosci. 2012;32:6177-6182.

45. Svec LA, Licht KM, Wade J. Pair bonding in the female zebra finch: a potential role for the nucleus taeniae. Neuroscience. 2009;160:275-283.

46. Fujii TG, Ikebuchi M, Okanoya K. Auditory responses to vocal sounds in the songbird nucleus taeniae of the amygdala and the adjacent arcopallium. Brain Behav Evol. 2016;87:275-289.

47. Dziuk PJ, Cook B. Passage of steroids through silicone rubber. Endocrinology. 1966;78:208-211.

48. Svec LA, Wade J. Estradiol induces region-specific inhibition of ZENK but does not affect the behavioral preference for tutored song in adult female zebra finches. Behav Brain Res. 2009;199:298-306.

49. Mello CV, Clayton DF. Song-induced ZENK gene expression in auditory pathways of songbird brain and its relation to the song control system. J Neurosci. 1994;14:6652-6666.

50. Replogle K, Arnold AP, Ball GF, et al. The Songbird Neurogenomics (SoNG) Initiative: community-based tools and strategies for study of brain gene function and evolution. BMC Genom. 2008;9:131.

51. Stokes TM, Leonard CM, Nottebohm F. The telencephalon, diencephalon, and mesencephalon of the canary, Serinus canaria, in stereotaxic coordinates. J Comp Neurol. 1974;156:337-374.

52. Elie JE, Soula HA, Trouvé C, Mathevon N, Vignal C. Housing conditions and sacrifice protocol affect neural activity and vocal behavior in a songbird species, the zebra finch (Taeniopygia guttata). C R Biol. 2015;338:825-837.

53. Maier T, Guell M, Serrano L. Correlation of mRNA and protein in complex biological samples. FEBS Lett. 2009;583:3966-3973.

54. Vogel C, Marcotte EM. Insights into the regulation of protein abundance from proteomic and transcriptomic analyses. Nat Rev Genet. 2012;13:227-232.

55. Sanford SE, Lange HS, Maney DL. Topography of estradiol-modulated genomic responses in the songbird auditory forebrain. Dev Neurobiol. 2010;70:73-86.

56. De Groof G, Balthazart J, Cornil CA, Van der Linden A. Topography and lateralized effect of acute aromatase inhibition on auditory processing in a seasonal songbird. J Neurosci. 2017;37:4243-4254.

57. Ball GF, Gentner TQ. They're playing our song: gene expression and birdsong perception. Neuron. 1998;21:271-274.

58. Nowicki S, Searcy WA. Song function and the evolution of female preferences: why birds sing, why brains matter. Ann NY Acad Sci. 2004;1016:704-723.

59. Yoder KM, Phan ML, Lu K, Vicario DS. He hears, she hears: are there sex differences in auditory processing? Dev Neurobiol. 2015;75:302-314.

60. Soma KK, Alday NA, Hau M, Schlinger BA. Dehydroepiandrosterone metabolism by 3beta-hydroxysteroid dehydrogenase/Delta5-Delta4 isomerase in adult zebra finch brain: sex difference and rapid effect of stress. Endocrinology. 2004;145:1668-1677.

How to cite this article: Lampen J, McAuley JD, Chang S-E, Wade J. ZENK induction in the zebra finch brain by song: Relationship to hemisphere, rhythm, oestradiol and sex. J Neuroendocrinol. 2017;29:e12543. https://doi.org/10.1111/jne.12543 